Research in Astron. Astrophys. Vol.0 (20xx) No.0, 000-000

http://www.raa-journal.org http://www.iop.org/journals/raa

Research in

(LTEX: RAA-2017-0254.tex; printed on October 10, 2018; 11:28)

$\boldsymbol{A}$ stronomy and

Astrophysics

\title{
Investigation of radio pulsar emission features using power spectra
}

\author{
V.M.Malofeev ${ }^{1}$ and S.A.Tyul'bashev ${ }^{1}$ \\ P.N.Lebedev Physical institute of the Russian Academy of Sciences, V.V.Vitkevich Pushchino \\ radioastronomy observatory, \\ PRAO ASC FIAN, Pushchino, Russia
}

\begin{abstract}
Since 2013 round-the-clock monitoring of the sky is carried out simultaneously in 96 space beams using the high-sensitivity radio telescope of LPA (Large Phased Array) at the frequency $110.25 \mathrm{MHz}$. These observations are made under the program of the interplanetary plasma investigation. The same data is used for the search for pulsars by means of the power spectra. For the increase of the pulsar search sensitivity the summation to 500-600 of power spectra corresponding to the different days of the observations is made. In the integrated spectra of the known pulsars, besides expected improvement of a signal-to-noise $(S / N)$ ratio for the frequency harmonics, were showed some features which are explored in the paper. We present 27 strongest pulsars which are in the field of declinations $21^{\circ}-42^{\circ}$ at which the connection of observable details in the integrated power spectra with the presence of pulsar periods of the second $\left(P_{2}\right)$ and third $\left(P_{3}\right)$ class has been discovered. The empirical relations for the calculation of these periods are obtained. The value $P_{2}$ is estimated for 26 pulsars, and for 15 sources it is made for the first time. The value $P_{3}$ is estimated for 13 pulsars, from them for 5 sources they are given for the first time.
\end{abstract}

Key words: pulsars: general - pulsars

\section{INTRODUCTION}

Fourier power spectrum is a good tool for searching for frequency of the periodical processes, therefore it is no wonder that search for pulsars is made, as a rule, with the use of power spectra. The Fourier transform is made or for the correlation function, or the Fourier transform of a signal is raised to a square. After detection of the harmonics in a spectrum the integration of a signal is made with a period corresponding to the inverse ratio of the first harmonic frequency. Thus search for a dispersion measure is carried out, in a case of observations in the several frequency channels. As a result of averaging the mean profile of a pulsar is obtained, which is the narrowest for the "correct" dispersion measure. The recurring observations using the same telescope by which the candidates for pulsars are is found, in addition to other frequency, are even better than observations by other telescopes, and it is the reliable method to confirm the pulsar existence. With small variations such organization of operation on the pulsar search is accepted everywhere, see the last review of Barr et al. (2013). The power spectrum as the such method of the search for pulsars is only auxiliary means, and in the further operation on the pulsar investigation is not used, as a rule.

In 2013 there is begun a round-the-clock monitoring of the sky by the radio telescope LPA under the program of investigation of an interplanetary plasma, and especially coronal mass ejections (Shishov et al.2016); project "Space Weather"). Besides of this program the review data was used for the search for pulsars also (Tyul'bashev et al.2016, 2017); project "BSA-Analytics", http://bsa-analytics.prao.ru). 
As pulsars are the objects with the small flux densities by their search the main problem is a realization of the highest possible sensitivity of observations so that it was possible to find out extremely weak objects. Taking into account the daily monitoring as an obvious method of increasing of the sensitivity, the accumulation of the power spectra is made, corresponding to the coordinates of the same points in the sky. In such integrated spectra, expected improvement of a $S / N$ ratio for the frequency harmonics and the appearance more their numbers (up to 108 for PSR J0323+3944), the appearance of the several features demanding an explanation was unexpected. In this paper the analysis of the integrated spectra is carried out and the nature of two observable features of power spectra is considered.

\section{OBSERVATIONS AND DATA PROCESSING}

Observations were made using the Large Phased Array of the P.N.Lebedev Physical Institute at the centre frequency $110.25 \mathrm{MHz}$. LPA is the phased array constructed on the half-wave dipoles, the geometrical area is $72000 \mathrm{~m}^{2}$ and the effective area is about $45000 \mathrm{~m}^{2}$ in a direction to zenith in the configuration used by us. The size of one beam is approximately $0.5^{\circ} \times 1^{\circ}$. The antenna is the meridian instrument, therefore any direction can be observed only once a day on an extent approximately 7 minutes. The details, concerning the radio telescopes created on the basis of the antenna LPA, are given in the papers Shishov et al. (2016), Tyul'bashev et al. (2016). There are two basic modes of a data recording. The first mode is a record in the six frequency channels with the channel bandwidth of $430 \mathrm{kHz}$ and the data-sampling interval $0.1 \mathrm{~s}$. The second mode is a record in a 32-channel frequency mode with the bandwidth of the channel $78 \mathrm{kHz}$ and the data-sampling interval $12.5 \mathrm{~ms}$. Since August, 2014 both modes are used simultaneously during observations in 96 beams of the antenna, overlapping the declinations $-9^{\circ}<\delta<42^{\circ}$. Data presented in this paper were obtained on the declinations $21^{\circ}<\delta<42^{\circ}$, where we have the peak sensitivity of the radio telescope and the minimum of noises is observed.

Before a calculation a power spectrum, cleaning from the pulse interferences was made and a quantity of the noise level was estimated. If this level was too big, that is more than the threshold level specially calculated for the given direction, the further operation was not use. In practice it has appeared that approximately from 20 to $30 \%$ of all records are rejected by the primary search for pulsars. The part of this rejected data can be used further for checkout of the search results and, probably, the pulsar investigations. The power spectra were under construction both for the 6-channel "small", and for the 32-channel "big" data. To obtain the Fourier power spectra fast Fourier transform was used. Temporary the file got out in length of 2048 points for "small" and 16384 points for "big" data, what was approximately half of the temporal beam of LPA. Further the spectra obtained independently for each frequency channel, were sum up and used for a primary search for the periods of new pulsars (Tyul'bashev et al.2017). The following stage was a summation of the power spectra for the different days corresponding to one direction on celestial sphere. At the moment of a beginning of a processing with "big" data already two years of the observations was available. There were 500-600 on overage files with the individual spectra to obtain the integrated power spectrum. That was expected 22-24 times increasing of the $S / N$ ratio for the harmonics of such integrated spectrum.

What it would be possible to expect from such summation? As radiation of the majority of pulsars exteriorly is represented a set of delta functions and in the obtained power spectrum on an exit there should be a set of delta functions. In practice a series of observations is limited on time and consequently an average power spectrum the harmonics should be built on the height, where the first harmonic one is the strongest. In real, such pattern is broken for an individual power spectra, as pulses of pulsars have different intensity, up to lack of a part of pulses more often. Frequently unsuccessful subtraction of a base line in the initial data and the strong low-frequency noise lead to that $\mathrm{S} / \mathrm{N}$ ratio in the first harmonic is lower, than in the second one.

As a result of summation of an individual power spectra we expected to see a certain "ideal" spectrum. However, effects of averaging have appeared absolutely unexpected almost for all pulsars of our list. So for 26 from 27 pulsars a modulation of amplitudes of harmonics with the quasi-periods from tens to hundreds milliseconds is observed. In fig.1 the example of an integrated (a) and individual (b) power spectrum of the pulsar J0528+2200 in the frequency interval to $40 \mathrm{~Hz}$ or $25 \mathrm{~ms}$ is given. 

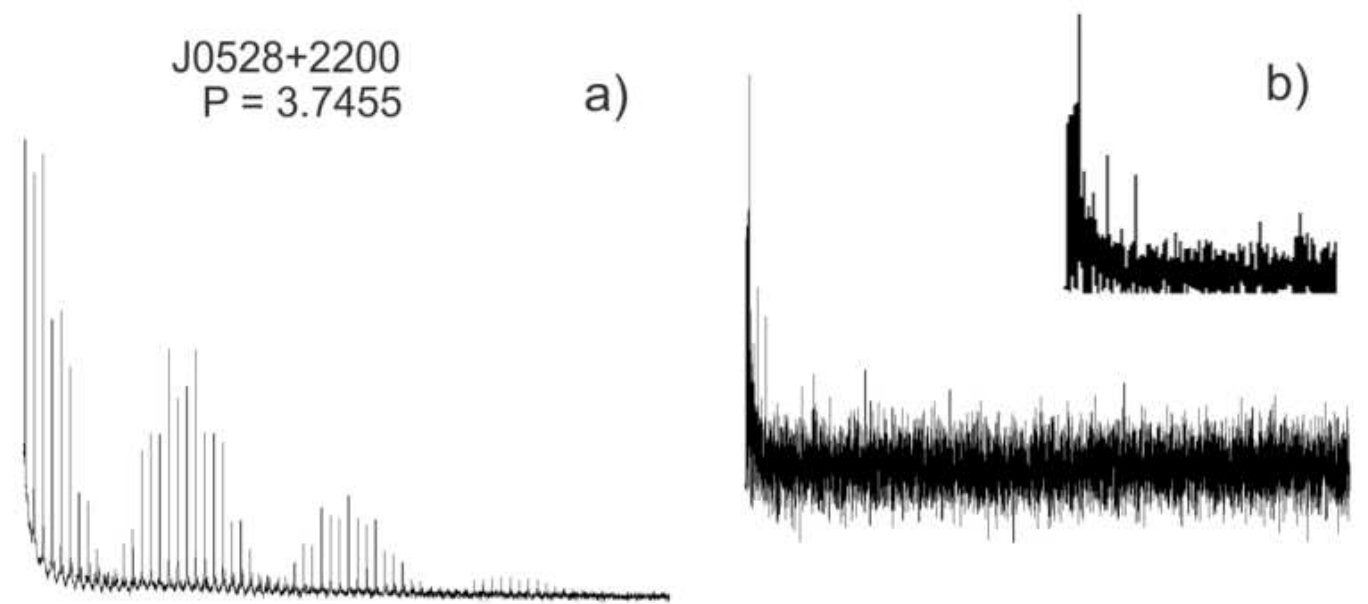

Fig. 1: Example of an integrated (a) and individual (b) power spectrum of pulsar J0528+2200. On insert fig. $1 \mathrm{~b}$ a fragment of a spectrum for the best representation of the first harmonics is given

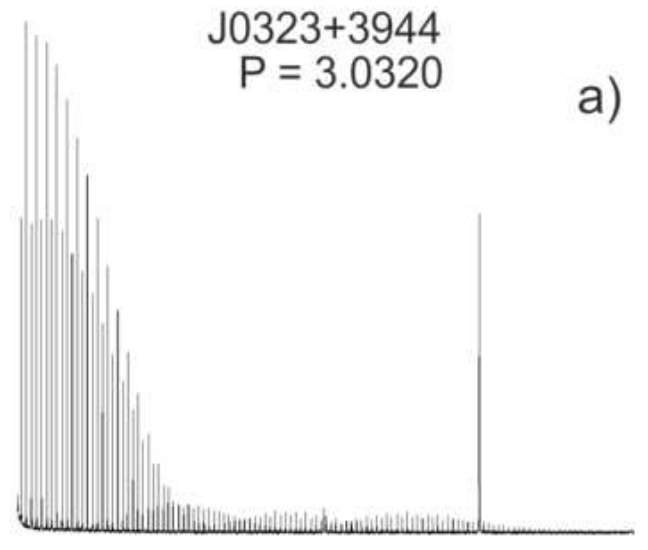

a)

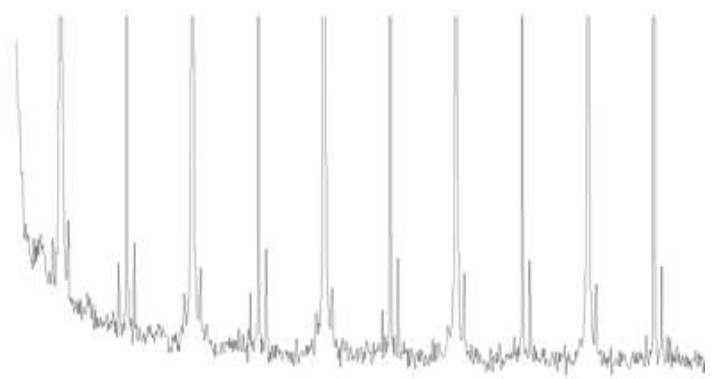

Fig. 2: The integrated power spectrum of pulsar J0323+3944. On a spectrum regularly appearing interferences of a man made origin (50 and $33 \mathrm{~ms}$ ) are showed; b) a fragment from a pulsar spectrum - first ten harmonics. The satellites of first harmonics are visible

From fig. 1 it is visible that the individual spectrum does not show any features. In an integrated spectrum a wavy structure is obviously expressed. A modulation showing periodic increasing of a $S / N$ ratio at far harmonics is presented. The pulsar is constantly visible practically during the most part of days of the monitoring. Its flux density at $400 \mathrm{MHz}$ according to catalogue ATNF is $S_{400}=57 \mathrm{mJy}$, and at the frequency of $102 \mathrm{MHz} S_{102}=100 \mathrm{mJy}$ (Malofeev et al.2000).

By detail consideration of the integrated power spectra, especially the first harmonics, the second feature has been found out. In fig.2a the integrated power spectrum of pulsar J0323+3944, and also a part of this spectrum in the large scale, including 10 first harmonics (fig.2b), is presented. The harmonics satellites near to pulsar main harmonic companions are visible clearly. Earlier these details in the power spectra were not mention by nobody. 
In fig.2a it is visible that the harmonics amplitudes have obviously wavy structure. For the first harmonics there is an obvious slope of power in harmonics, and the modulation, as in fig.1a, but less expressed is visible. In fig. $2 b$ it is well visible that near to the harmonics, there are the harmonics satellites which are not multiple to a pulsar period. One of these satellites is to the left, and another to the right of a main harmonic, but distance to the left and to the right satellites is not identical. It is interesting that both satellites are visible not in all pulsars, one of satellites is more often visible only. Presence of such details is observed in 13 pulsars from 27 ones.

The power spectra of 25 pulsars are presented in fig.3, and 2 more pulsars are in fig.1, 2. Figures are prepared so that on an axis of the frequencies all were limited till the presence of the visible harmonics. Therefore the extreme right frequency on all power spectra is the different. The value of a period given together with the name of a pulsar, allows be oriented in frequency domain, as the frequency of the first harmonic is equal to the quantity return to a rotating period of a pulsar. Continuation of fig. 3 is fig.4 showing the fragments of the power spectra of several pulsars with clearly visible harmonics satellites, except of pulsar J0323+3944 presented in fig. 2 b.

Not all spectra in fig.3 and fig.4 show an identical collection of the details given in fig.1-2, but all have any typical features. The analysis of the integrated spectra is made in the following paragraph. 


\section{INVESTIGATION OF POWER SPECTRA}

As it is known, besides a rotation period $\left(P_{1}\right)$ in some of pulsars other periodic or quasi-periodic features which are not related directly to a main period are observed also. It is, so-called, "the drifting subpulse phenomenon" which is characterized by two periods $P_{2}$ and $P_{3}$. At the very beginning of pulsar investigation it was discovered (Drake \& Craft 1968) that there were pulsars with inner structure of an individual pulse - subpulses which showed the regular drift of a phase of arrival inside the so-called "pulsar window", forming "the drift bands" on the diagram: the number of a period (axis of ordinates) a phase of a period (abscissa axis). The $P_{2}$ is accepted to term as a period of the second class. The value $P_{2}$ is the horizontal drift band separation in time units. A period of the third class $\left(P_{3}\right)$, this distance is determined in number of periods $P_{1}$ on an axis of ordinates. Drift to the back end of a "window" terms as positive, and to the forward one as negative. One of the first pulsars with the very regular and bright drift (PSR J0814+7429) has been found out in Pushchino Vitkevich \& Shitov (1970). Now the drift of subpulses is known in about 70 pulsars and, approximately, for the same number $P_{2}$ and $P_{3}$ are measured (Weltevrede et al. 2006).

There are some models which are attempt to explain the drift effect. The most known: not radial pulsations of a neutron star Ruderman (1968), the sparking gap model over a polar cap Ruderman \& Sutherland (1975), rotating of a radiation pattern around a magnetic axis Sieber \& Oster (1975), developed in "a rotating carousel" model Deshpande \& Rankin (1999), and its application to PSR B0943+10 Rankin et al. (2003), and, at last, a feedback model is proposed by Wright (2003). Unfortunately, not any of models does not explain all variety of the details related to the effect of subpulse drifts.

We have tried to explain the features showed in fig.1-4 in the integrated power spectra by the presence of the $P_{2}$ and the $P_{3}$. We take, for an example, two pulsars J0528+2200 (fig.1) and J0323+3944 (fig.2), which, according to the investigation of Weltevrede et al. (2006) have these periods. This paper, containing the most measurements of the drifting subpulse phenomenon, was taken as basic for the comparison with our data. It presents the result of detailed investigations of 187 pulsars at the frequency of $1420 \mathrm{MHz}$ spent in Westerbork (Netherlands). The one-dimensional and the two-dimensional Fourier power spectra in a pulse window have been used, for measuring as the modulation coefficient, and $P_{2}$ and $P_{3}$. In this analysis the records containing of some thousands of the pulses were used, as a rule. In fig. $1 \mathrm{a}$ it is visible that the middle or a maximum of the first "hump" of modulation in a power spectrum is necessary on a harmonic under the number 19 that corresponds to a period $200 \mathrm{~ms}$. According to Weltevrede et al. (2006) in this pulsar a $P_{2}$ is or $-200 \mathrm{~ms}$, or $+500 \mathrm{~ms}$. Our estimate allows select the single-valued of a period $200 \mathrm{~ms}$. We cannot estimate a sign of the drift. Thus, the period of the second class is calculated from a simple relation:

$$
P_{2}=P_{1} / n,
$$

where $n$ it is the harmonic number at which the middle of the first "hump" of modulation in an average power spectrum takes place, and $P_{1}$ is a rotation pulsar period. Analyzing the known periods of the third class of some pulsars, we have obtained the empirical formulas for these satellites of the main harmonic:

$$
\begin{aligned}
& P_{l}(n)=P_{1}(n)\left(n \times \frac{P_{3}}{\left(n \times P_{3}-1\right)}\right) \\
& P_{r}(n)=P_{1}(n)\left(n \times \frac{P_{3}}{\left(n \times P_{3}+1\right)}\right),
\end{aligned}
$$

where $P_{l}$ is a period of the left satellite, $P_{r}$ is a period of the right one and $P_{3}$ is a period of the third class, in terms of $P_{1}$. It is follows from relations (2) that:

$$
\begin{aligned}
& P_{3}=P_{l}(n) \times \frac{1}{n \times \Delta P_{l}(n)} \\
& P_{3}=P_{r}(n) \times \frac{1}{n \times \Delta P_{r}(n)},
\end{aligned}
$$

where $\Delta P_{l}(n)=P_{l}(n)-P_{1}(n)$, and $\Delta P_{r}(n)=P_{1}(n)-P_{r}(n)$. Presence of the harmonics satellites in the spectrum, apparently, reflects the presence of the beating of two periodic processes with 


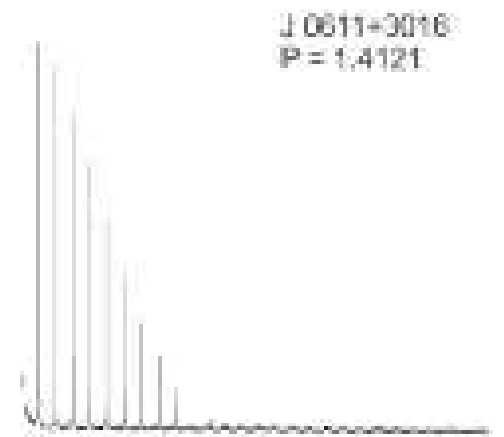

J0928+3037

$\mathrm{P}=2.0019$

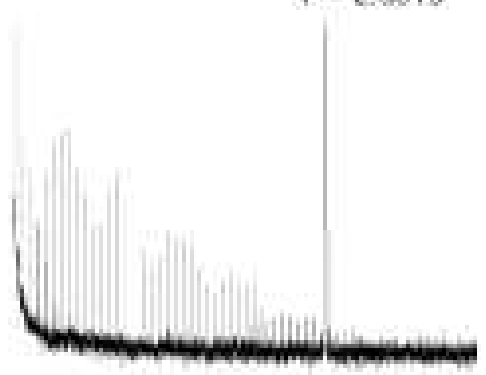

$11532+2745$

$P=1.1248$

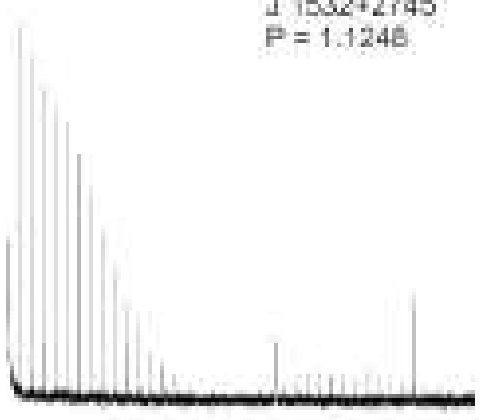

J176e+3034

$P=0.9473$

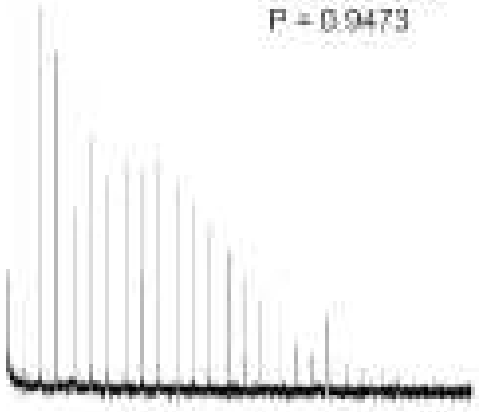

J $1912+2525$

$P=0.6214$

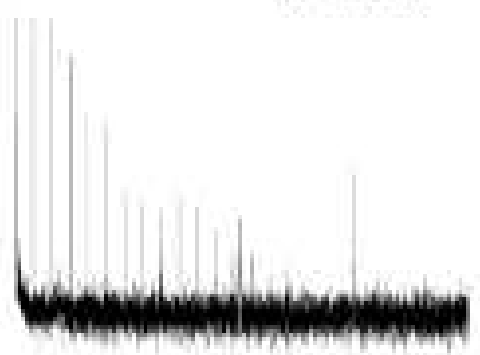

I. $0613+3721$

$P=0.2979$
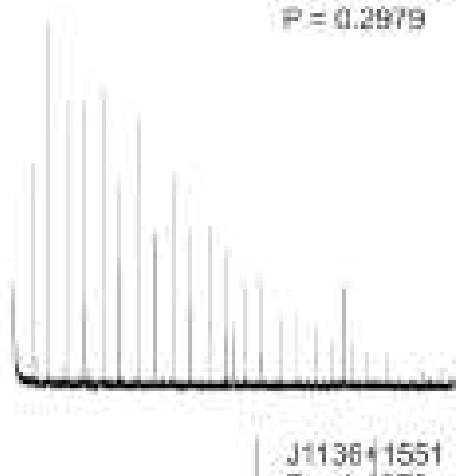

$P=1.1679$

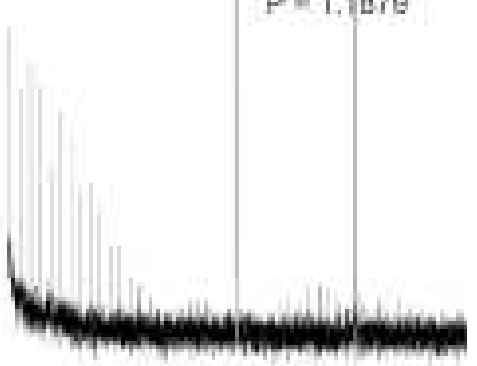

J) $1635+2418$

$P=0.4905$

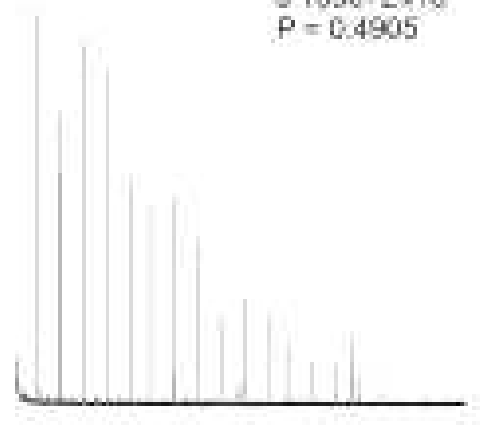

J $1813+4012$

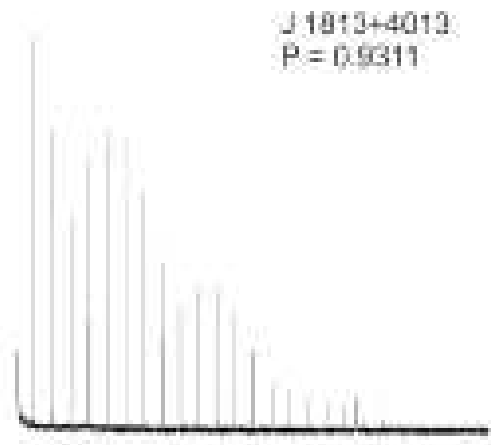

J $1421+2153$

$P=1.3373$

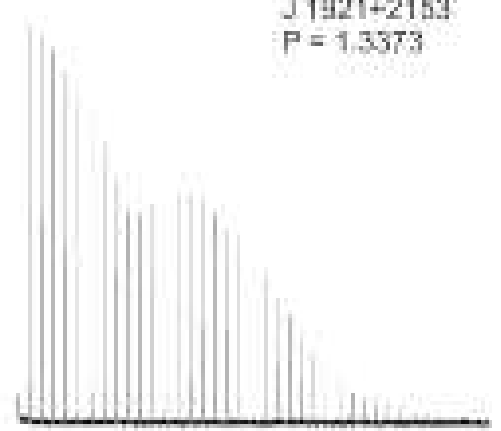

$40028+26.37$

$p=10.507$

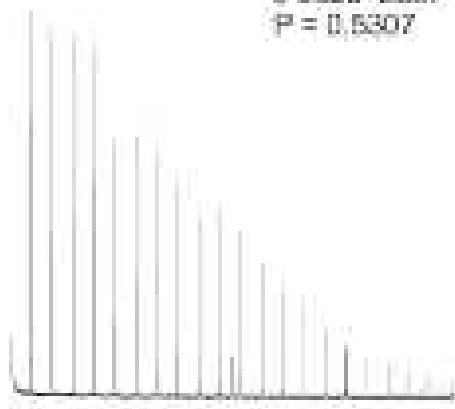

J $1239+2463$

$\rho=1.3824$

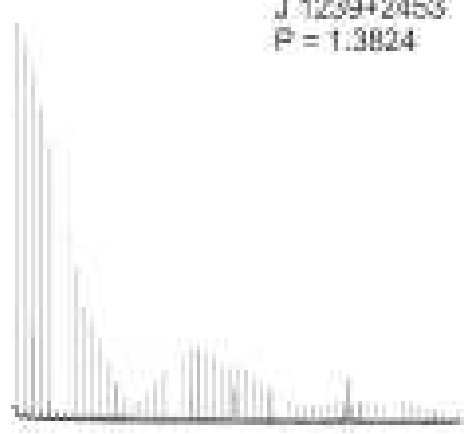

$41741+2750$

$p=0.360 \mathrm{~V}$
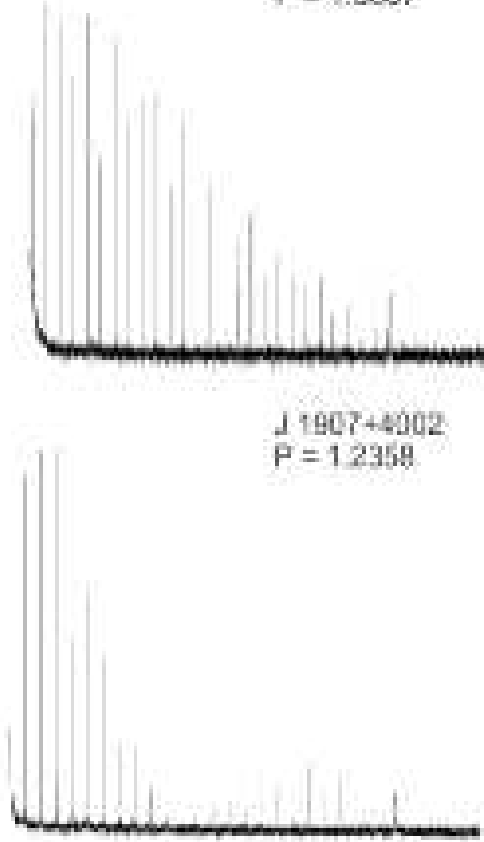

$J 2018+2839$

$P=0.5579$

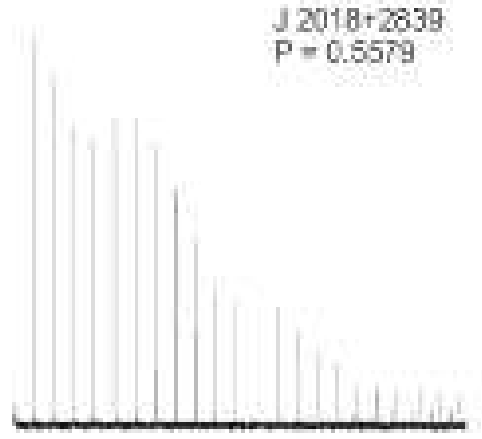



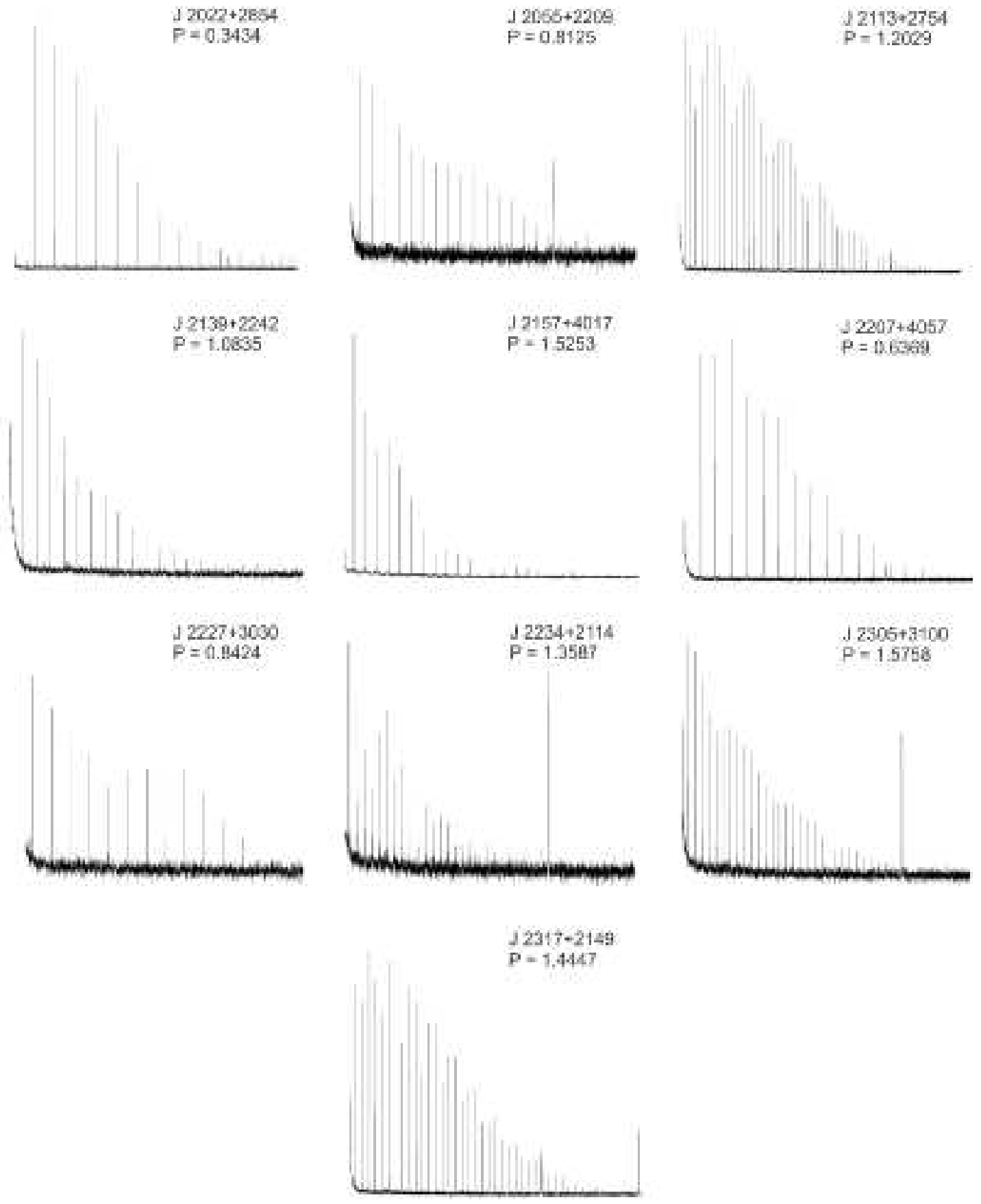

Fig. 3: Integrated power spectra of 25 pulsars

period $P_{1}$ and $P_{3}$. It is necessary to notice that both satellites are not in all pulsars, and in a case of presence both, value of $P_{3}$ calculated from equation (3), is slightly more in the left companion (by $7-20 \%$ ), but, as a rule, both values coincide within a measuring error. It is necessary to note that in the paper of Weltevrede et al. (2006) also there are the pulsars with the double value not only $P_{2}$, but also 

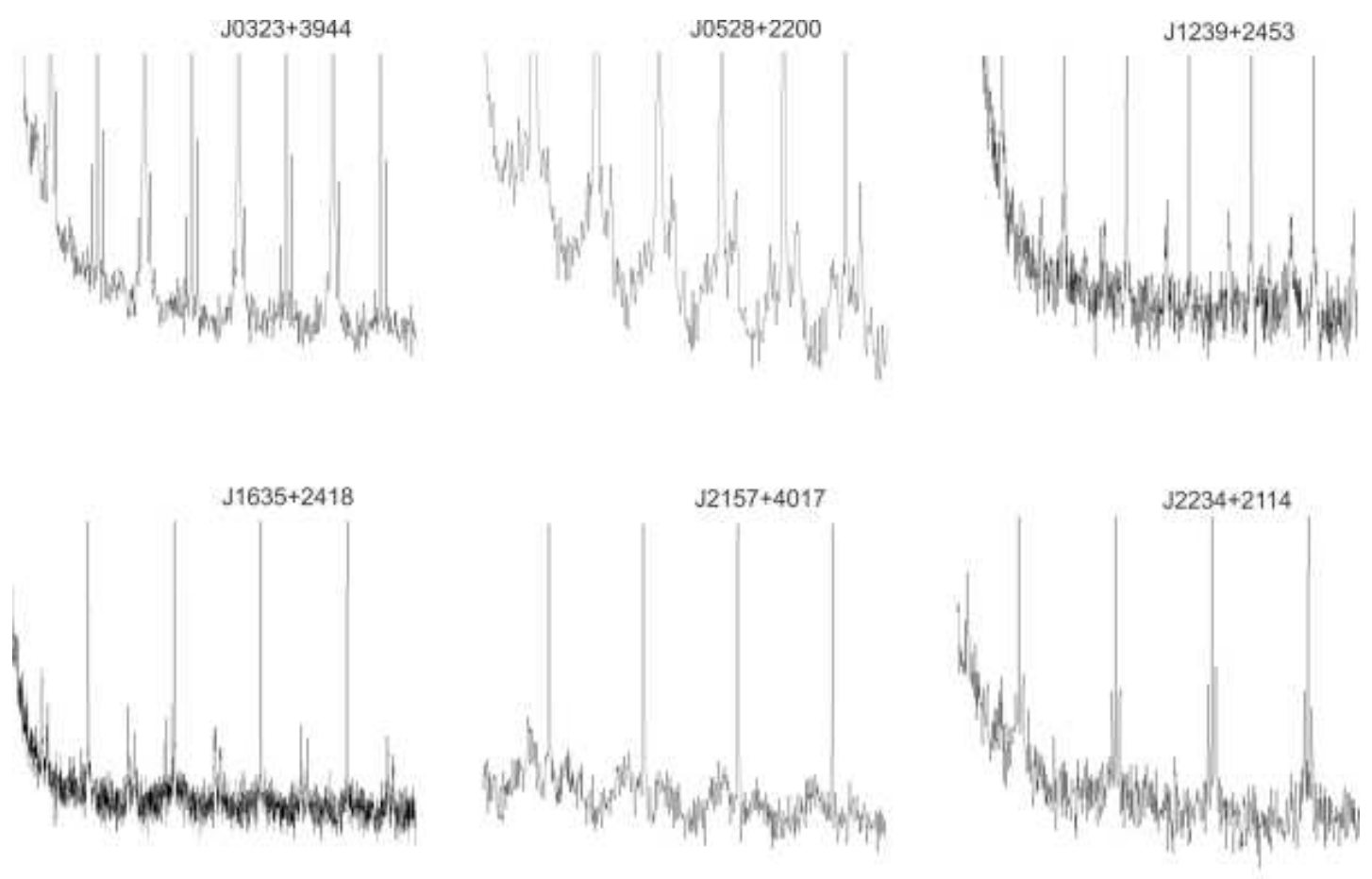

Fig. 4: The fragments of the integrated power spectra of pulsars are presented, with the most expressed harmonics satellites, except of pulsar J0528+2200 which is in fig. $1 \mathrm{~b}$

$P_{3}$. We will return to the our example. Having calculated distance to the left and the right satellites at several harmonics of pulsar J0323+3944 (fig.2b), according to the equation (3), we calculated the period $P_{3}=8.4 \pm 1.3$. Weltevrede et al. (2006) give the same quantity $P_{3}=8.4 \pm 0.1$.

In table 1 the information on 14 pulsars from our sample is presented. These pulsars are common with the list of the objects of Weltevrede et al. (2006). In the first column of the table names of the pulsars in $\mathrm{J} 2000$ are given. We give in the second column a pulsar period from the ATNF catalogue, in the third column period $P_{2}$, and in the fifth column period $P_{3}$ of the pulsars, measured in our observations. For comparison in the columns four and six the data from the paper Weltevrede et al. (2006) are given. The errors of the measuring $P_{2}$ are related to an accuracy of the definition of the number of a harmonic at which the middle of the first maximum of the amplitude modulation is appeared. The error of its definition, as a rule, is \pm 0.5 numbers of a harmonic of the basic spectrum. The error of a measuring $P_{3}$ is related to the dispersion of the values $P_{l}(n)$ and $P_{r}(n)$, measured, not less than, for the three satellites at the several first harmonics. As a rule, the number of the measures made from 6 to 20 . The first measurements of $P_{2}$ and $P_{3}$ for 13 pulsars are presented in tab.2. These pulsars have not included into the paper Weltevrede et al. (2006). In the first, second and third columns the same quantities, as in tab. 1 are given, and in the fourth one values $P_{3}$ are presented. $P_{3}$ given in tab.1,2, obtained in the main, as the mean value between $P_{l}(n)$ and $P_{r}(n)$.

The comparison of our estimates of the periods $P_{2}$ and $P_{3}$ with data of Weltevrede et al. (2006) shows their good agreement. Moreover, on our estimates of period $P_{2}$, for some pulsars, it is possible to remove of the not single-valued in a direction of a subpulses motion in a pulse window. Our accuracy of an evaluation of $P_{2}$ in most cases is high, and for $P_{3}$ we see the comparable values. 
Table 1: Estimates of the periods $P_{2}$ and $P_{3}$ from Fourier power spectra and according to Weltevrede et al. (2006)

\begin{tabular}{|c|c|c|c|c|c|}
\hline name & $\begin{array}{l}P_{1} \\
(\mathrm{~s})\end{array}$ & $\begin{array}{c}P_{2(L P A)} \\
\text { (ms) }\end{array}$ & $\begin{array}{c}P_{2(W)} \\
(\mathrm{ms})\end{array}$ & $\overline{P_{3(L P A)}}$ & $P_{3(W)}$ \\
\hline $\mathrm{J} 0323+3944$ & 3.03 & $112 \pm 12$ & $152_{-25}^{+42}$ & $8.4 \pm 1.3$ & $8.4 \pm 0.1$ \\
\hline \multirow[t]{2}{*}{$\mathrm{J} 0528+2200$} & 3.75 & $200 \pm 10$ & $-208_{-90}^{+20}$ & $4.9 \pm 0.5$ & $3.8 \pm 0.7$ \\
\hline & & & $520_{-105}^{+580}$ & & $3.7 \pm 0.4$ \\
\hline J0826+2637 & 0.53 & $150 \pm 25$ & $80_{-10}^{+60}$ & $5.7 \pm 0.4$ & $7 \pm 2$ \\
\hline \multirow[t]{2}{*}{$\mathrm{J} 1136+1551$} & 1.19 & $400 \pm 70$ & $430_{-50}^{+400}$ & & $3 \pm 1$ \\
\hline & & & $660_{-300}^{+180}$ & & \\
\hline \multirow[t]{2}{*}{$\mathrm{J} 1239+2453$} & 1.38 & $60 \pm 3$ & $61_{-9}^{+4}$ & $2.7 \pm 0.1$ & $2.7 \pm 0.1$ \\
\hline & & & $77_{-11}^{+5}$ & & \\
\hline $\mathrm{J} 1813+4013$ & 0.93 & $170 \pm 15$ & & & \\
\hline \multirow[t]{2}{*}{$\mathrm{J} 1907+4002$} & 1.24 & $65 \pm 4$ & & & \\
\hline & & $250 \pm 50$ & & & \\
\hline \multirow[t]{2}{*}{$\mathrm{J} 1921+2153$} & 1.34 & $33 \pm 1$ & $13_{-1}^{+1}$ & $4.2 \pm 0.5$ & $4.4 \pm 0.1$ \\
\hline & & $100 \pm 3$ & $41 \pm 4$ & & \\
\hline \multirow[t]{2}{*}{$\mathrm{J} 2018+2839$} & 0.56 & & $-19_{-12}^{+2}$ & $4.1 \pm 0.4$ & $4 \pm 4$ \\
\hline & & $93 \pm 8$ & $-108_{-44}^{+23}$ & & \\
\hline \multirow[t]{2}{*}{$\mathrm{J} 2022+2854$} & 0.34 & $26 \pm 3$ & $24_{-2}^{+14}$ & & $2.3 \pm 0.1$ \\
\hline & & & $-52_{-14}^{+5}$ & & $2.5 \pm 0.2$ \\
\hline $\mathrm{J} 2113+2754$ & 1.2 & $200 \pm 15$ & $470_{-50}^{+60}$ & & $4.4 \pm 0.1$ \\
\hline $\mathrm{J} 2157+4017$ & 1.53 & $300 \pm 40$ & $470_{-45}^{+370}$ & $4.5 \pm 0.7$ & $3.1 \pm 0.8$ \\
\hline $\mathrm{J} 2305+3100$ & 1.58 & $190 \pm 30$ & $66_{-1}^{+14}$ & $2.2 \pm 0.4$ & $2.1 \pm 0.1$ \\
\hline $\mathrm{J} 2317+2149$ & 1.44 & $470 \pm 30$ & & & \\
\hline
\end{tabular}

\section{SUMMARY AND DISCUSSION}

The investigations of the integrated Fourier power spectra became possible, because there was data on four-year monitoring of the large part of a sky. The volume of the data has exceeded already 100 terabyte. The continuous time of an accumulation for each point in the sky, after rejection of the data with the interferences, exceeds two days. This rich data has given already a series of interesting results on the solar wind investigation (Chashei et al.2015, Shishov et al.2016), on the search for pulsars (Tyul'bashev et al. 2016, Tyul'bashev et al. 2017) and on the search for the prompt radio transients (Tyul'bashev \& Tyul'bashev 2017a). The integrated power spectra reveal also two new, unexpected features which we interpret as a display of the periodical processes in the field of radiation of the pulsar pulses.

If our interpretation is true for all 14 pulsars in tab. 1 we have obtained an estimate of $P_{2}$. For 7 pulsars it well agree with the data given by Weltevrede et al. (2006), and for 4 sources there is a discrepancy in the measurements. Thus for three pulsars from these four $(\mathrm{J} 1921+2153$, J2157+4070 and $\mathbf{J} 2305+3103$ ) values of $P_{3}$ are coincide with Weltevrede et al. (2006). Only for J2113+2754 we 
Table 2: Estimates of $P_{2}$ and $P_{3}$ for the pulsars which have not included into the paper Weltevrede et al. (2006)

\begin{tabular}{cccc}
\hline name & $P_{1}(\mathrm{~s})$ & $P_{2(L P A)}(\mathrm{ms})$ & $P_{3(L P A)}$ \\
\hline $\mathrm{J} 0611+3016$ & 1.41 & & $2.5 \pm 0,2$ \\
$\mathrm{~J} 0613+3722$ & 0.29 & $180 \pm 70$ & \\
$\mathrm{~J} 0928+3037$ & 2.09 & $280 \pm 20$ & $4.8 \pm 0,5$ \\
$\mathrm{~J} 1532+2745$ & 1.12 & $39 \pm 2$ & $4.6 \pm 0,5$ \\
$\mathrm{~J} 1635+2418$ & 0.49 & $140 \pm 30$ & $2.0 \pm 0,15$ \\
$\mathrm{~J} 1741+2758$ & 1.36 & $270_{-60}^{+120}$ & \\
& & & \\
$\mathrm{~J} 1758+3030$ & 0.95 & $105 \pm 15$ & \\
$\mathrm{~J} 1912+2525$ & 0.62 & $70 \pm 8$ & \\
$\mathrm{~J} 2055+2209$ & 0.81 & $80 \pm 10$ & \\
$\mathrm{~J} 2139+2242$ & 1.08 & $160 \pm 20$ & \\
$\mathrm{~J} 2207+4057$ & 0.63 & $210 \pm 40$ & \\
$\mathrm{~J} 2227+3030$ & 0.84 & $105 \pm 15$ & \\
$\mathrm{~J} 2234+2114$ & 1.38 & $230 \pm 20$ & $23.3 \pm 3,5$ \\
\hline
\end{tabular}

do not see the satellites at the first harmonics. For two pulsars we cannot exactly determinate the first "hump" on a spectrum. Therefore for pulsars J1907+4002 and J1921+2153 we give two values of this quantity (Tab.1). For 3 pulsars (J1813+4013, J1907+4002 and J2317+2149) we made for the first time the estimate of $P_{2}$, because in the paper Weltevrede et al. (2006) there are no this data. Such situation can indicate that or the additional investigations of these pulsars are necessary, which do not have agreement, or the nature of the modulations which we observe is related not only to a period $P_{2}$. The values of $P_{3}$ has confirmed for 8 pulsars from 11 of list of Weltevrede et al. (2006), and in three remained sources we do not see already the significant harmonics of the satellites in the integrated power spectrum (tab.1). The data for 13 pulsars, given in tab. 2 are new and they give estimates of $P_{2}$ for 12 sources and estimates of $P_{3}$ for 5 pulsars, including pulsar J0928+3037 recently discovered by us (Tyul'bashev et al. 2016). The values $P_{2}$ are in a range 26-470 $\mathrm{ms}$ and the values $P_{3}$ lie in a range from 2 to $23.3 P_{1}$ (Tab.1-2). All new estimates of both $P_{2}$ and $P_{3}$ need in the confirmation by other methods.

Thus, as a result of the analysis of the integrated power spectra obtained by a summation of 500-600 daily spectra, their some new features are discovered. It is shown that two of them, namely a modulation with the periods from 3 to 40 first harmonics is reflected to a period of the second class $\left(P_{2}\right)$, and a presence of the harmonic satellites is testified of a period of the third class $\left(P_{3}\right)$. We find the empirical relations (1-3) for the calculation of both periods. As a result of the analysis, a measuring of these periods and a comparison with the data of (Weltevrede et al. 2006) the value $P_{2}$ is estimated for 26 pulsars, and for 15 sources it is made for the first time. The value $P_{3}$ is estimated for 13 pulsars, from them for 5 sources they are given for the first time.

\section{ACKNOWLEDGEMENTS}

The authors are grateful for support of the grant of the Russian Foundation for Basic Research 1602-00954 and for an active participation of V.S.Tyulbashev, V.V.Oreshko and S.V.Logvinenko. Authors express gratitude L.B.Potapova for the help with the paper design and I.F.Malov for the help with the translation.

\section{References}

Barr E.D. et al., 2013, MNRAS, 435, 2234

Chashei I.V., Shishov V.I., Tyul'bashev S.A., Subaev I.A., Oreshko V.V., Logvinenko S.V., 2015, Sol. Phys., 290, 2577

Catalogue ATNF - http://www.atnf.siro.au

Deshpande A.A. \& Rankin J.M., 1999, ApJ, 524, 1008 
Drake F.D. \& Craft H.D., 1968, Nature, 220, 231

Malofeev V.M., Malov O.I., Schegoleva N.V., 2000, Astron. Rep., 44, 436

Sieber W. \& Oster L., 1975, A\&A, 38, 325

Rankin J.M., Suleymanova S.A., Deshpande A.A., 2003, MNRAS, 340, 1076

Ruderman M.A., 1968, Nature, 218, 1128

Ruderman M.A. \& Sutherland P.G., 1975, ApJ, 196, 51

Shishov V.I., et al., 2016, Astron. Rep., 60, 1067

Tyul'bashev S.A., Tyul'bashev V.S., Oreshko V.V., Logvinenko S.V., 2016, Astron. Rep., 60, 220

Tyul'bashev S.A. et al., 2017, Astron. Rep., 61, 848

Tyul'bashev S.A., Tyul'bashev V.S., 2017a, ATsir., 1636, 1

Vitkevich V.V. \& Shitov Yu.P., 1970, Nature, 225, 248

Weltevrede P., Edvards R.T., Stappers B.W., 2006, A\&A, 445, 243

Wright G.A.E., 2003, MNRAS, 344, 1041

This paper was prepared with the RAA LATEX macro v1.2. 